\title{
Roots of Unity Associated to Strongly Detected Boundary Slopes
}

\author{
Srikanth Kuppum and Xingru Zhang ${ }^{1}$
}

\section{Introduction}

Let $M$ be a knot manifold, i.e. a compact connected irreducible orientable 3 -manifold whose boundary is a torus. Let $R(M)$ denote the $S L_{2}(\mathbb{C})$ representation variety of $M$, and $X(M)$ the character variety of $M$. Recall that the character $\chi_{\rho} \in X(M)$ of a representation $\rho \in R(M)$ is the complex valued function $\chi_{\rho}: \pi_{1}(M) \rightarrow \mathbb{C}$ defined by $\chi_{\rho}(\gamma)=\operatorname{trace}(\rho(\gamma))$ for $\gamma \in \pi_{1}(M)$. Each element $\gamma \in \pi_{1}(M)$ defines a regular function $\tau_{\gamma}$ on $X(M)$ by $\tau_{\gamma}\left(\chi_{\rho}\right)=\operatorname{trace}(\rho(\gamma))$, called the trace function defined by $\gamma$. Let $X_{0}$ be an irreducible algebraic curve in $X(M)$ and let $\tilde{X}_{0}$ be the smooth projective completion of $X_{0}$. Note that each trace function $\tau_{\gamma}$ extends to a rational function $\tilde{\tau}_{\gamma}$ on $\tilde{X}_{0}$. By the fundamental theorem of [CS], if $x \in \tilde{X}_{0}$ is a pole of some trace function $\tilde{\tau}_{\gamma}$, then there is an essential surface $S$ in $M$ associated to $x$ via an action of $\pi_{1}(M)$ on a Bass-Serre simplicial tree (Note that the set of poles of all trace functions is equal to the set of ideal points in $\tilde{X}_{0}$ ). If in addition the element $\gamma$ is a peripheral element of $\pi_{1}(M)$, then any essential surface $S$ associated to a pole $x$ of $\tilde{\tau}_{\gamma}$ must have non-empty boundary on $\partial M$. In such case, the boundary slope of $S$ on the torus $\partial M$ is said to be strongly detected by the ideal point $x$ of $\tilde{X}_{0}$. Moreover the trace function of the primitive element $\beta \in \pi_{1}(\partial M)$ (or its inverse) corresponding to the $\partial$-slope of $S$ has finite value at the ideal point $x$. It was proved in [CCGLS] that $\tilde{\tau}_{\beta}(x)=\lambda+\lambda^{-1}$ for some root of unity $\lambda$. The order of $\lambda$ has geometric significance; it divides the number of boundary components of any non-closed component of a reduced essential surface associated to $x$. (An essential surface in $M$ associated to the ideal point $x$ is called reduced if it has the minimal number of boundary components among all essential surfaces associated to $x$.) We call a root of unity arising this way as associated to a strongly detected boundary slope of $M$.

Most of known examples of roots of unity associated to strongly detected boundary slopes are \pm 1 . For instance, this is always the case for the exterior of any 2-bridge knot in $S^{3}$ (this result is implicitly contained in [O]). In [D], several examples of roots of unity of order four and six were given. The main purpose of this paper is to produce an infinite family of knot manifolds each of which has a root of unity of order four associated to a strongly detected boundary slope.

Let $L=K_{1} \cup K_{2}$ be the Whitehead link in $S^{3}$ shown in Figure 1 (a), and let $W$ be

\footnotetext{
${ }^{1}$ Partially supported by NSF grant DMS 0204428.
} 


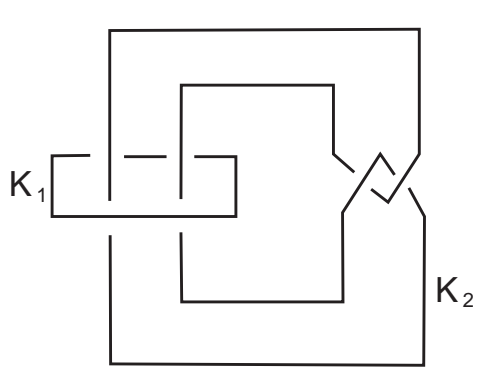

(a)

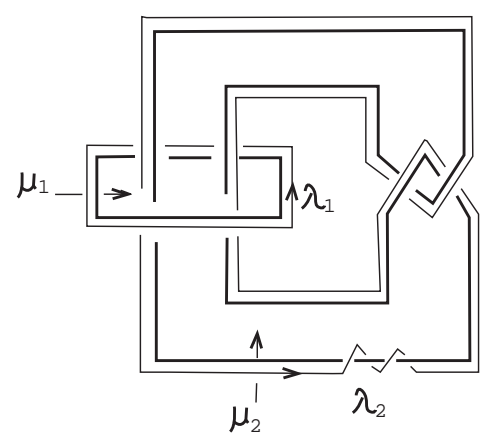

(b)

Figure 1: The Whitehead link $K_{1} \cup K_{2}$, the two oriented meridians $\mu_{1}, \mu_{2}$, and the two oriented longitudes $\lambda_{1}, \lambda_{2}$.

the exterior of $L$. Let $T_{i}$ be the component of $\partial W$ corresponding to $K_{i}, i=1,2$. The slopes on each of the tori $T_{i}$ will be perameterized with respect to the standard meridianlongitude basis of $K_{i}$ (when considered as a knot in $S^{3}$ ). The orientations of the meridians and longitudes are chosen as shown in Figure 1 (b).

Theorem 1 Let $M_{n}$ be the manifold obtained by Dehn filling $W$ along $T_{1}$ with integer slope $n$. Then for each integer $k \geq 1$, the slope $1 / k$ on $\partial M_{4 k+2}=T_{2}$ is a strongly detected boundary slope to which a root of unity of order four is associated.

To prove Theorem 1, we need recall some more results from [CCGLS]. Let $M$ be a knot manifold. Fix a basis $\mathcal{B}=\{\mu, \lambda\}$ for $\pi_{1}(\partial M)$. The set of slopes in the boundary torus $\partial M$ is parameterized with respect to the basis $\mathcal{B}$ as $\{m / n ; m, n \in \mathbb{Z},(m, n)=1\}$ where $m$ is the $\mu$-coordinate and $n$ is the $\lambda$-coordinate, i.e. a slope $m / n$ corresponds to the inverse pair $\mu^{m} \lambda^{n}$ and $\mu^{-m} \lambda^{-n}$ in $\pi_{1}(\partial M)$. For the pair $(M, \mathcal{B})$, a two variable polynomial $A(x, y)=\sum a_{i j} x^{i} y^{j} \in \mathbb{Z}[x, y]$, called the $A$-polynomial of the pair $(M, \mathcal{B})$, can be uniquely defined up to sign [CCGLS]. The Newton polygon of $A(x, y)$ is the convex hull of the set $\left\{(i, j) ; a_{i j} \neq 0\right\}$ in the real $x y$-plane. Let $E$ be an edge of the Newton polygon of slope $n / m$ in the $x y$-plane, and let $\Theta_{E}(z)$ be the single variable polynomial defined by

$$
\Theta_{E}(z)= \begin{cases}\sum_{(i, j) \in E} a_{i j} z^{i}, & \text { if } m \neq 0 \\ \sum_{(i, j) \in E} a_{i j} z^{j}, & \text { if } m=0\end{cases}
$$

$\Theta_{E}(z)$ was called the edge polynomial of $E$ in [CCGLS]. The following properties concerning edge polynomials were proved in [CCGLS]:

(1) Each non-zero root of an edge polynomial $\Theta_{E}(z)$ is a root of unity;

(2) If $n / m$ is the slope of an edge $E$ of the Newton polygon in the $x y$-plane, then $m / n$ is a strongly detected boundary slope of $M$; 
(3) If $E$ is an edge of the Newton polygon with slope $n / m$, then for every non-zero root $c$ of the edge polynomial $\Theta_{E}(z)$, there is an irreducible curve $X_{0}$ in $X(M)$ and an ideal point $x$ in $\tilde{X}_{0}$ such that $\tilde{\tau}_{\beta}(x)=c^{m}+c^{-m}$ (if $m \neq 0$ ) or $\tau_{\beta}(x)=c+c^{-1}$ (if $m=0$ ), where $\beta=\mu^{m} \lambda^{n}$, and the order of $c^{m}$ (if $m \neq 0$ ) or $c$ (if $m=0$ ) divides the number of boundary components of any component of a reduced essential surface associated to $x$.

Note that part of property (3) is implicitly contained in [CCGLS].

Therefore to prove Theorem 1, we only need to show that the Newton polygon of the $A$-polynomial of $M_{4 k+2}$ has an edge of slope $k$ whose edge polynomial has a root of unity of order 4 , for each $k>0$.

Note that each $M_{n}$ is a punctured torus bundle with monodromy $\left(\begin{array}{cc}-n+2 & 1 \\ -1 & 0\end{array}\right)$ (see [HMW, Proposition 3], note that the slopes given in [HMW] differ from ours by sign). So $M_{n}$ is hyperbolic for each $n>4$. Incompressible surfaces in hyperbolic punctured torus bundles are classified in [FH] and [CJR]. According to the algorithm given in [FH], $M_{4 k+2}$ has two boundary slopes, 4 and $1 / k$, besides the boundary slope 0 of a fiber (note that $M_{n}$ has the $L R$-decomposition $-R L^{n-4}$, and that the slopes given in $[\mathrm{FH}]$ are the reciprocal of ours). For any hyperbolic knot manifold $M$, the Newton polygon of the $A$-polynomial of $M$ contains at least two edges with different slopes corresponding to strict boundary slopes of $M$. It follows that $k$ must be the slope of an edge of the Newton polygon of the $A$-polynomial of $M_{4 k+2}$. Also note that by [FH], the boundary slope $1 / k$ in $\partial M_{4 k+2}$ bounds an incompressible surface in $M$ with genus $\frac{k}{2}-1$ and with exactly four boundary components.

In Section 2, we try to obtain the $A$-polynomial of $M_{4 k+2}$ formally as far as possible following the algorithm prescribed in [CCGLS]. Nevertheless the calculation is stuck in the last step where a resultant is fairly difficult to calculate explicitly. However at that stage we are able to calculate the edge polynomial for the edge of slope $k$ without knowing the final explicit expression of the $A$-polynomial. This part of the calculation of the edge polynomial will be carried out in Section 4 . On the way to get the $A$-polynomial of $M_{4 k+2}$, we also show (and need to show) that the representation variety of $M_{4 k+2}$ has exactly one irreducible component containing irreducible representations of $\pi_{1}\left(M_{4 k+2}\right)$. This is done in Section 3.

\section{Getting the $A$-polynomial of $M_{4 k+2}$}

Consider the Whitehead link shown in the figure above. Let $\mu_{1}, \mu_{2}$ be the meridians of the two components shown in the figure. We take these two meridians as members of generators needed in the Wirtinger presentation of $\pi_{1}(W)$. The Wirtinger presentation can be easily 
simplified to the following form:

$$
\pi_{1}(W)=<\mu_{1}, \mu_{2} ; \mu_{1} \mu_{2} \mu_{1}^{-1} \mu_{2}^{-1} \mu_{1} \mu_{2}^{-1} \mu_{1}^{-1} \mu_{2}=\mu_{2} \mu_{1}^{-1} \mu_{2}^{-1} \mu_{1} \mu_{2}^{-1} \mu_{1}^{-1} \mu_{2} \mu_{1}>.
$$

The longitude $\lambda_{1}$ (see Figure $1(\mathrm{~b})$ ) of the component $K_{1}$ of the Whitehead link can be expressed as

$$
\lambda_{1}=\mu_{1}^{-1} \mu_{2}^{-1} \mu_{1} \mu_{2} \mu_{1}^{-1} \mu_{2} \mu_{1} \mu_{2}^{-1}
$$

and the longitude $\lambda_{2}$ for $K_{2}$ is given by

$$
\lambda_{2}=\mu_{2}^{-1} \mu_{1}^{-1} \mu_{2} \mu_{1} \mu_{2}^{-1} \mu_{1} \mu_{2} \mu_{1}^{-1}
$$

(cf. [CL, proof of Lemma 9.4].)

The Dehn filling of $W$ along $T_{1}$ with the slope $n$ gives an additional relation $\lambda_{1} \mu_{1}^{n}=1$. So the fundamental group of $M_{n}$ has a presentation:

$$
\begin{gathered}
\pi_{1}\left(M_{n}\right)=<\mu_{2}, \mu_{1} ; \quad \mu_{1} \mu_{2} \mu_{1}^{-1} \mu_{2}^{-1} \mu_{1} \mu_{2}^{-1} \mu_{1}^{-1} \mu_{2}=\mu_{2} \mu_{1}^{-1} \mu_{2}^{-1} \mu_{1} \mu_{2}^{-1} \mu_{1}^{-1} \mu_{2} \mu_{1}, \\
\mu_{1}^{-1} \mu_{2}^{-1} \mu_{1} \mu_{2} \mu_{1}^{-1} \mu_{2} \mu_{1} \mu_{2}^{-1} \mu_{1}^{n}=1>.
\end{gathered}
$$

Any irreducible representation $\rho$ of $\pi_{1}(W)$ can be conjugated so that

$$
\rho\left(\mu_{1}\right)=\left(\begin{array}{cc}
M & 0 \\
t & M^{-1}
\end{array}\right), \rho\left(\mu_{2}\right)=\left(\begin{array}{cc}
p & 1 \\
0 & p^{-1}
\end{array}\right) .
$$

Substituting the above matrices in the relation of $\pi_{1}(W)$ given above, we get several defining polynomials which all have the following polynomial $F(M, p, t)$ as the only common factor $F(M, p, t)=-M p+M^{3} p+M p^{3}-M^{3} p^{3}+M^{2} t-M^{4} t+p^{2} t-4 M^{2} p^{2} t+M^{4} p^{2} t-p^{4} t+$ $M^{2} p^{4} t-M p t^{2}+2 M^{3} p t^{2}+2 M p^{3} t^{2}-M^{3} p^{3} t^{2}-M^{2} p^{2} t^{3}$.

Let $\mathcal{R}(W)$ be the variety defined by this polynomial. Then the points $(M, p, t)$ in $\mathcal{R}(W)$ with $M \neq 0, p \neq 0, t \neq 0$ is the set of irreducible representations of $\pi_{1}(W)$ modulo the conjugation. (Note that the variety defined by the polynomial $F$ is the same as the variety defined by all the polynomials involved from the matrix equation. This follows from a dimension counting argument. We omit the details.)

Next we calculate such variety $\mathcal{R}\left(M_{n}\right)$ for $M_{n}, n>4$ even. Considering the second relation of $\pi_{1}\left(M_{n}\right)$, we calculate $\rho\left(\lambda_{1}\right), \rho\left(\mu_{1}^{n}\right)$ and then $\rho\left(\lambda_{1} \mu_{1}^{n}\right)$. The $(2,2)$-entry of $\rho\left(\lambda_{1} \mu_{1}^{n}\right)$ minus 1 is the polynomial

$$
\begin{aligned}
& 1 /\left(M^{2} p^{2} M^{n}\right)\left(M^{2} p^{2}+M p t-2 M^{3} p t-2 M p^{3} t+3 M^{3} p^{3} t+M p^{5} t-M^{3} p^{5} t-M^{2} t^{2}+M^{4} t^{2}-\right. \\
& p^{2} t^{2}+5 M^{2} p^{2} t^{2}-2 M^{4} p^{2} t^{2}+p^{4} t^{2}-3 M^{2} p^{4} t^{2}+M^{4} p^{4} t^{2}+M p t^{3}-2 M^{3} p t^{3}-2 M p^{3} t^{3}+2 M^{3} p^{3} t^{3}+ \\
& \left.M^{2} p^{2} t^{4}\right)-1
\end{aligned}
$$

Multiply this polynomial by $M^{2} p^{2} M^{n}$ and call the resulting polynomial $G$. We have 
$G(M, p, t)=M^{2} p^{2}+M p t-2 M^{3} p t-2 M p^{3} t+3 M^{3} p^{3} t+M p^{5} t-M^{3} p^{5} t-M^{2} t^{2}+M^{4} t^{2}-p^{2} t^{2}+$ $5 M^{2} p^{2} t^{2}-2 M^{4} p^{2} t^{2}+p^{4} t^{2}-3 M^{2} p^{4} t^{2}+M^{4} p^{4} t^{2}+M p t^{3}-2 M^{3} p t^{3}-2 M p^{3} t^{3}+2 M^{3} p^{3} t^{3}+$ $M^{2} p^{2} t^{4}-M^{2} p^{2} M^{n}$.

This polynomial depends on the surgery slope $n$. The resultant of $F$ and $G$ eliminating the variable $t$ should contain a factor which defines $\mathcal{R}\left(M_{n}\right)$. The resultant is

$$
\begin{aligned}
& -M^{2 n}-M^{2+n}+M^{4+n}+M^{2+2 n}-M^{4} p^{2}+M^{n} p^{2}+M^{3 n} p^{2}-2 M^{2+n} p^{2}+2 M^{2+2 n} p^{2}- \\
& M^{4+2 n} p^{2}-M^{2 n} p^{4}-M^{2+n} p^{4}+M^{4+n} p^{4}+M^{2+2 n} p^{4} .
\end{aligned}
$$

When $n>4$ is even, this polynomial can be factored as

$M^{4}(M-1)(M+1)\left[\left(M^{n-2}+M^{2 n-4}\right)+\left(\delta_{n}+M^{n-2} \delta_{n}-M^{n-4}-M^{2 n-2}\right) p^{2}+\left(M^{n-2}+M^{2 n-4}\right) p^{4}\right]$,

where $\delta_{n}=\frac{M^{2 n-2}-1}{M^{2}-1}=1+M^{2}+M^{4}+M^{6}+\cdots+M^{2 n-4}$.

We shall prove that the factor

$$
U(M, p)=\left(M^{n-2}+M^{2 n-4}\right)+\left(\delta_{n}+M^{n-2} \delta_{n}-M^{n-4}-M^{2 n-2}\right) p^{2}+\left(M^{n-2}+M^{2 n-4}\right) p^{4}
$$

is irreducible over $\mathbb{C}$ for all $n=4 k+2 \geq 6$, and thus the variety defined by $U$ is irreducible for all $n=4 k+2 \geq 6$. It also follows that the variety defined by the polynomial $U$ is $\mathcal{R}\left(M_{n}\right)$.

Our primary interest is the family of manifolds $M_{4 k+2}$ and for the rest of the article, we assume that $n=4 k+2$ and $k \geq 1$.

To get the $A$-polynomial of $M_{4 k+2}$ formally, we take the $(1,1)$-entry $\rho\left(\lambda_{2}\right)$ minus the new variable $q$, and call the resulting polynomial $H$ (up to a multiple of a power of $M$ and a power of $p$ ).

$$
\begin{aligned}
& H(M, p, t, q)=-M^{3} p^{2}+p t-3 M^{2} p t+2 M^{4} p t-p^{3} t+2 M^{2} p^{3} t-M^{4} p^{3} t-M t^{2} \\
& \quad+2 M^{3} t^{2}-M^{5} t^{2}+3 M p^{2} t^{2}-5 M^{3} p^{2} t^{2}+M^{5} p^{2} t^{2}-M p^{4} t^{2}+M^{3} p^{4} t^{2}-2 M^{2} p t^{3} \\
& \quad+2 M^{4} p t^{3}+2 M^{2} p^{3} t^{3}-M^{4} p^{3} t^{3}-M^{3} p^{2} t^{4}+q M^{3} p^{2} .
\end{aligned}
$$

Let $V$ be the resultant of $F$ and $H$ eliminating the variable $t$, then

$$
\begin{aligned}
& V(M, p, q)=M^{4} p^{4} q^{2}+2 M^{2} p^{2} q+p^{2} q+M^{2} q^{2}-M^{4} p^{2} q^{2}+M^{2}+M^{4} p^{2} q-q-p^{2} q^{2} \\
& \quad+p^{4} q^{2}-M^{2} p^{4} q-M^{2} p^{4} q^{3}-2 M^{2} p^{2} q^{2}-M^{4} q .
\end{aligned}
$$

Finally the resultant of $U$ and $V$ eliminating the variable $M$ should contain a factor which is the A-polynomial of $M_{n}$ in the variables $p$ and $q$ (recall that generically $p$ and $q$ are eigenvalues of $\rho\left(\mu_{2}\right)$ and $\rho\left(\lambda_{2}\right)$ respectively). However this last resultant seems pretty hard to calculate explicitly. Even if we were able to calculate the resultant, to determine if the resulting polynomial is irreducible and then further determine which factor is the $A$ polynomial would be another two difficult tasks. For our purpose, we get around this 
difficulty by calculating directly the relevant edge polynomial for $M_{n}$ when $n=4 k+2$, from the formal expression of the resultant of $U$ and $V$. This will be done in Section 4 .

\section{$3 \quad$ Irreducibility of $\mathcal{R}\left(M_{4 k+2}\right)$}

Recall that we assume $n=4 k+2$ and $k \geq 1$. The variety $\mathcal{R}\left(M_{n}\right)$, is defined by the single two variable polynomial

$$
U(M, p)=\left(M^{n-2}+M^{2 n-4}\right)+\left(\delta_{n}+M^{n-2} \delta_{n}-M^{n-4}-M^{2 n-2}\right) p^{2}+\left(M^{n-2}+M^{2 n-4}\right) p^{4}
$$

where $\delta_{n}=\frac{M^{2 n-2}-1}{M^{2}-1}$. In this section we show that $\mathcal{R}\left(M_{4 k+2}\right)$ is an irreducible variety. We only need to show that $U$ is an irreducible polynomial over $\mathbb{C}$.

Firstly, we observe that there is no non-trivial factorization of $U=f(M) \cdot g(M, p)$ where $f(M)$ is a non-constant polynomial in $M$ and $g(M, p)$ is a quartic when viewed as a polynomial in $p$ with coefficients from $\mathbb{C}[M]$. If there were such a factorization, then $f(M)$ would be a common factor of the coefficients of $p^{0}, p^{2}$ and $p^{4}$ in $U(M, p)$ given above. The coefficient of $p^{0}$ ( or equivalently $p^{4}$ ) is given by $\left(M^{n-2}+M^{2 n-4}\right)=M^{n-2}\left(M^{n-2}+1\right)$. But neither zero nor any root of $M^{n-2}+1$ is a root of $\left(\delta_{n}+M^{n-2} \delta_{n}-M^{n-4}-M^{2 n-2}\right)$, the coefficient of $p^{2}$ in $U(M, p)$.

So the irreducibility of $U$ is a consequence of the above observation and of Lemma 2 and Lemma 3 given below, where it is demonstrated that the polynomial $U$ cannot be factored either as a product of a linear factor and a cubic factor or as a product of two quadratic factors when viewed as polynomials in $p$.

Lemma 2 The polynomial $U$ cannot be factored as $f(p) g(p)$ where $f(p)=A+B p$ and $g(p)=C+D p+E p^{2}+F p^{3}$, for $A, B, C, D, E$ and $F$ polynomials in $M$ with complex coefficients.

Proof. Suppose otherwise that the polynomial $U$ can be factored as $f(p) g(p)$ as described in the lemma. Then $B \neq 0$ and $F \neq 0$. We may assume that $A \neq 0$ and $C \neq 0$ as $p$ does not divide $U$. Multiplying out $f(p) g(p)$ and comparing terms with those of $U$, we get

(1) $A C=M^{n-2}+M^{2 n-4}$

(2) $A D+B C=0$

(3) $A E+B D=\delta_{n}+M^{n-2} \delta_{n}-M^{n-4}-M^{2 n-2}$

(4) $A F+B E=0$

(5) $B F=M^{n-2}+M^{2 n-4}$.

We claim that $D \neq 0$ and $E \neq 0$. For, if $D=0$, from (2) we would have $B C=0$, but this contradicts $B \neq 0, C \neq 0$. And if $E=0$, from (4) we would have $A F=0$ which contradicts 
$A \neq 0, F \neq 0$. With this claim none of the coefficients $A, B, C, D, E$ or $F$ can be zero. From (2) and (4), we get

(6) $\frac{A}{B}=-\frac{C}{D}=-\frac{E}{F}=\lambda$ (say), a rational polynomial in $M$.

From (1) and (5), we get $A C=B F$. Together with (6), we get

(7) $F=-\lambda^{2} D$.

From (3) (6) and (7) we have

(8) $B D\left(\lambda^{4}+1\right)=\delta_{n}+M^{n-2} \delta_{n}-M^{n-4}-M^{2 n-2}$

From (1) and (6) we have

(9) $-\lambda^{2} B D=M^{n-2}+M^{2 n-4}$.

From (8) and (9) we have $-\frac{\delta_{n}+M^{n-2} \delta_{n}-M^{n-4}-M^{2 n-2}}{M^{n-2}+M^{2 n-4}}=\frac{\lambda^{4}+1}{\lambda^{2}}=\left(\lambda+\frac{1}{\lambda}\right)^{2}-2$,

$\frac{\text { i.e. }\left(M^{n-2}+M^{2 n-4}\right)-\left(\delta_{n}+M^{n-2} \delta_{n}-M^{n-4}-M^{2 n-2}\right)}{M^{n-2}+M^{2 n-4}}=\left(\lambda+\frac{1}{\lambda}\right)^{2}=\frac{2 L-H}{L}$,

where $L=M^{n-2}+M^{2 n-4}$ and $H=\delta_{n}+M^{n-2} \delta_{n}-M^{n-4}-M^{2 n-2}$. Since $\lambda$ is a rational polynomial in $M, \frac{2 L-H}{L}$ must be a perfect square of a rational polynomial in $\mathbb{C}(M)$. However, $L$ and $H$ are relatively prime (no common roots in $\mathbb{C}$ ) which implies $2 L-H$ and $L$ are relatively prime. Hence, $L=\left(M^{n-2}+M^{2 n-4}\right)$ must be the square of a polynomial in $\mathbb{C}[M]$. Now, $\left(M^{n-2}+M^{2 n-4}\right)=M^{n-2}\left(M^{n-2}+1\right)$ where the first factor $M^{n-2}=M^{4 k}$ is a perfect square but the other factor $M^{n-2}+1$ is not a perfect square as it has $n-2$ distinct roots of negative unity. $\diamond$

Lemma 3 The polynomial $U$ cannot be factored as a product $f(p) g(p)$ where $f(p)=A+$ $B p+C p^{2}$ and $g(p)=D+E p+F p^{2}$ for $A, B, C, D, E$ and $F$, polynomials in $M$ over complex numbers.

Proof. As in Lemma 2, we may assume $A \neq 0, C \neq 0, D \neq 0, F \neq 0$. Multiplying out the polynomials $f$ and $g$ and comparing terms with those of $U$, we have :

(1) $A D=M^{n-2}+M^{2 n-4}$

(2) $A E+B D=0$

(3) $A F+B E+C D=\delta_{n}+M^{n-2} \delta_{n}-M^{n-4}-M^{2 n-2}$

(4) $C E+B F=0$

(5) $C F=M^{n-2}+M^{2 n-4}$

The proof is broken into the cases : $E=0$ and $E \neq 0$.

$\underline{\text { Case } 1}: E=0$.

From (2), we get $B=0$. So (3) becomes

(6) $A F+C D=\delta_{n}+M^{n-2} \delta_{n}-M^{n-4}-M^{2 n-2}$

Using (1) and (5) in (6), we have

$\left(\frac{M^{n-2}+M^{2 n-4}}{D}\right) F+\left(\frac{M^{n-2}+M^{2 n-4}}{F}\right) D=\delta_{n}+M^{n-2} \delta_{n}-M^{n-4}-M^{2 n-2}$. Set $\frac{F}{D}=\lambda, \mathrm{a}$ 
rational polynomial in $M$.

Then $\lambda+\frac{1}{\lambda}=\frac{\delta_{n}+M^{n-2} \delta_{n}-M^{n-4}-M^{2 n-2}}{M^{n-2}+M^{2 n-4}}$.

Denote the numerator above as $H=\delta_{n}+M^{n-2} \delta_{n}-M^{n-4}-M^{2 n-2}$ and the denominator as $L=M^{n-2}+M^{2 n-4}$. Solving the above equation $\lambda+\frac{1}{\lambda}=\frac{H}{L}$,

$$
\lambda=\frac{H \pm \sqrt{(H+2 L)(H-2 L)}}{2 L}
$$

Since $\lambda$ is a rational polynomial in $M,(H+2 L)(H-2 L)$ should be a perfect square of a polynomial in $\mathbb{C}[M]$. Clearly, $L$ and $H$ are relatively prime (no common root over $\mathbb{C}$ ), which implies $H+2 L$ and $H-2 L$ are relatively prime. Hence it is necessary that each of $H+2 L$ and $H-2 L$ be a perfect square as a polynomial in $M$. But by Lemma 4 , this is not true.

Case 2: $E \neq 0$.

It follows from (2) that $B \neq 0$. From (2) and (4) we have

(7) $\frac{A}{D}=-\frac{B}{E}=\frac{C}{F}=\lambda$ (say), a rational in $M$.

From (1),(5) and (7), we get $\lambda D^{2}=\lambda F^{2}$. Hence $D^{2}=F^{2}$ (as $\lambda \neq 0$, else $A=0$ from (7)).

So $D= \pm F$. This leads to the following two subcases :

Subcase 2a $: D=F$;

Subcase $2 \mathrm{~b}: D=-F$.

In Subcase 2a, we have $A=\lambda F=C$ from (7). Now from (1) and (7) we have

(8) $\lambda=\frac{\left(M^{n-2}+M^{2 n-4}\right)}{D^{2}}$

Using (7) and (8) in (3), we get

(9) $\left(M^{n-2}+M^{2 n-4}\right)\left(\frac{F}{D}\right)-\left(M^{n-2}+M^{2 n-4}\right)\left(\frac{E}{D}\right)^{2}+\left(M^{n-2}+M^{2 n-4}\right)\left(\frac{F}{D}\right)$

$$
=\left(\delta_{n}+M^{n-2} \delta_{n}-M^{n-4}-M^{2 n-2}\right) .
$$

Now (9) would imply

$$
\begin{aligned}
\frac{E^{2}}{D^{2}}= & \left(\frac{E}{D}\right)^{2}=2-\frac{\delta_{n}+M^{n-2} \delta_{n}-M^{n-4}-M^{2 n-2}}{\left(M^{n-2}+M^{2 n-4}\right)} \\
& =\frac{2\left(M^{n-2}+M^{2 n-4}\right)-\left(\delta_{n}+M^{n-2} \delta_{n}-M^{n-4}-M^{2 n-2}\right)}{\left(M^{n-2}+M^{2 n-4}\right)},
\end{aligned}
$$

which must be the square of a rational in $\mathbb{C}(M)$. However, $\left(M^{n-2}+M^{2 n-4}\right)$ and $\left(\delta_{n}+\right.$ $\left.M^{n-2} \delta_{n}-M^{n-4}-M^{2 n-2}\right)$ are relatively prime and hence $\left(M^{n-2}+M^{2 n-4}\right)$ and $2\left(M^{n-2}+\right.$ $\left.M^{2 n-4}\right)-\left(\delta_{n}+M^{n-2} \delta_{n}-M^{n-4}-M^{2 n-2}\right)$ are relatively prime. So $\left(M^{n-2}+M^{2 n-4}\right)$ must be a square of a polynomial in $M$, which is not true as seen in the proof of previous lemma.

Similarly in Subcase $2 \mathrm{~b}$, we would get

$\frac{E^{2}}{D^{2}}=\frac{-2\left(M^{n-2}+M^{2 n-4}\right)-\left(\delta_{n}+M^{n-2} \delta_{n}-M^{n-4}-M^{2 n-2}\right)}{\left(M^{n-2}+M^{2 n-4}\right)}$

and as in Subcase $2 \mathrm{a},\left(M^{n-2}+M^{2 n-4}\right)$ would be a perfect square, which is not true as before. $\diamond$ 
Lemma 4 For a given $n,\left(\delta_{n}+M^{n-2} \delta_{n}-M^{n-4}-M^{2 n-2}\right)+2\left(M^{n-2}+M^{2 n-4}\right)$ and $\left(\delta_{n}+\right.$ $\left.M^{n-2} \delta_{n}-M^{n-4}-M^{2 n-2}\right)-2\left(M^{n-2}+M^{2 n-4}\right)$ cannot both be perfect squares in $\mathbb{C}[M]$.

Proof. For $k=1$,

$$
\begin{aligned}
\left(\delta_{n}\right. & \left.+M^{n-2} \delta_{n}-M^{n-4}-M^{2 n-2}\right)+2\left(M^{n-2}+M^{2 n-4}\right) \\
& =1+4 M^{4}+2 M^{6}+4 M^{8}+M^{12} \\
& =\left(M^{2}-M+1\right)\left(M^{2}+M+1\right)\left(M^{4}-M^{3}+M+1\right)\left(M^{4}+M^{3}-M+1\right)
\end{aligned}
$$

has a primitive cube root of unity as an unrepeated root and hence is not a perfect square.

For $k>1$,

$$
\begin{aligned}
\left(\delta_{n}+\right. & \left.M^{n-2} \delta_{n}-M^{n-4}-M^{2 n-2}\right)-2\left(M^{n-2}+M^{2 n-4}\right)= \\
= & \left(1+M^{2}+M^{4}+\cdots+M^{4 k-4}\right)+2 M^{4 k+2}\left(1+M^{2}+M^{4}+\cdots+M^{4 k-4}\right)+ \\
& \quad+2 M^{4 k+2}+M^{8 k+4}\left(1+M^{2}+M^{4}+\cdots+M^{4 k-4}\right)= \\
= & \left(1+M^{2}+M^{4}+\cdots+M^{4 k-4}\right)\left(1+M^{4 k+2}\right)^{2}
\end{aligned}
$$

is a perfect square only if $\left(1+M^{2}+M^{4}+\cdots+M^{4 k-4}\right)=\frac{\left(M^{2}\right)^{2 k-1}-1}{M^{2}-1}$ is a perfect square. However every root of the latter polynomial is a root of $M^{4 k-2}-1=0$ which has distinct $(4 k-2)$ roots of unity. Thus, for $k>1,\left(\delta_{n}+M^{n-2} \delta_{n}-M^{n-4}-M^{2 n-2}\right)-2\left(M^{n-2}+M^{2 n-4}\right)$ is not a perfect square.

This completes the proof of the lemma. $\diamond$

\section{Edge polynomial Computations}

Recall from Section 2 we have polynomials

$V(M, p, q)=a+b M^{2}+c M^{4}$, where $a=p^{2} p+p^{4} q^{2}-q-p^{2} q^{2}$ while $b=-p^{4} q^{3}+q^{2}-$ $2 p^{2} q^{2}+2 p^{2} q-p^{4} q+1$, and

$$
\begin{aligned}
U(M, p, & q)=\left(M^{n-2}+M^{2 n-4}\right)+\left(\delta_{n}+M^{n-2} \delta_{n}-M^{n-4}-M^{2 n-2}\right) p^{2}+\left(M^{2 n-4}+M^{n-2}\right) p^{4} \\
= & \left.M^{n-2}+M^{2 n-4}\right)+\left(1+M^{2}+M^{4}+M^{6}+\cdots\left(M^{2}\right)^{n-2}+\right. \\
& \left.+M^{n-2}\left(1+M^{2}+M^{4}+M^{6}+\cdots\left(M^{2}\right)^{n-2}\right)-M^{n-4}-M^{2 n-2}\right) p^{2}+ \\
& +\left(M^{n-2}+M^{2 n-4}\right) p^{4} \\
= & p^{2}+p^{2} M^{2}+p^{2} M^{4}+\ldots+p^{2} M^{n-6}+ \\
& +(0) M^{n-4}+\left(1+2 p^{2}+p^{4}\right) M^{n-2}+\left(2 p^{2}\right) M^{n}+\left(2 p^{2}\right) M^{n+2}+\ldots+\left(2 p^{2}\right) M^{2 n-6}+ \\
& +\left(1+2 p^{2}+p^{4}\right) M^{2 n-4}+(0) M^{2 n-2}+p^{2} M^{2 n}+\ldots+p^{2} M^{3 n-6}
\end{aligned}
$$

and the A-polynomial of $M_{n}$ is a factor of the resultant of the two polynomials $U$ and $V$ eliminating the variable $M$. Actually we may simply treat $U$ and $V$ as polynomials in $M^{2}$, instead of $M$. So the A-polynomial of $M_{n}$ is a factor of the following determinant of the square matrix of size $\frac{3 n-6}{2}+\frac{4}{2}=\frac{3 n}{2}-1=6 k+2$ :(see next page) 


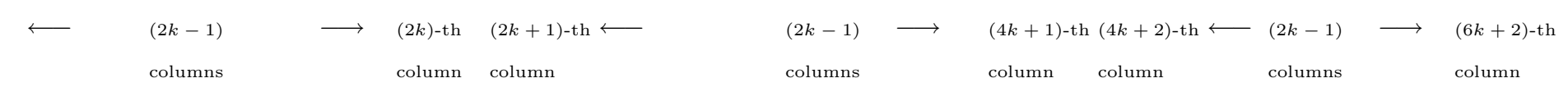

$\vec{\sigma}$

\begin{tabular}{|llllllllllllllllll}
$p^{2}$ & $p^{2}$ & $p^{2}$ & $\ldots$ & $p^{2}$ & 0 & $\sigma$ & $2 p^{2}$ & $2 p^{2}$ & $\ldots$ & $2 p^{2}$ & $\sigma$ & 0 & $p^{2}$ & $\ldots$ & $p^{2}$ & 0 \\
0 & $p^{2}$ & $p^{2}$ & $p^{2}$ & $\ldots$ & $p^{2}$ & 0 & $\sigma$ & $2 p^{2}$ & $2 p^{2}$ & $\ldots$ & $2 p^{2}$ & $\sigma$ & 0 & $p^{2}$ & $\ldots$ & $p^{2}$ \\
$a$ & $b$ & $a$ & 0 & 0 & $\ldots$ & 0 & 0 & 0 & 0 & 0 & $\ldots$ & 0 & 0 & 0 & $\ldots$ & 0 \\
0 & $a$ & $b$ & $a$ & 0 & $\ldots$ & $\ldots$ & $\ldots$ & $\ldots$ & $\ldots$ & $\ldots$ & $\ldots$ & $\ldots$ & $\ldots$ & $\ldots$ & $\ldots$ & 0 \\
0 & 0 & $a$ & $b$ & $a$ & 0 & $\ldots$ & $\ldots$ & $\ldots$ & $\ldots \ldots$ & $\ldots$ & $\ldots$ & $\ldots$ & $\ldots$ & $\ldots$ & $\ldots$ & 0 \\
0 & 0 & 0 & $a$ & $b$ & $a$ & 0 & $\ldots$ & $\ldots$ & $\ldots$ & $\ldots$ & $\ldots$ & $\ldots$ & $\ldots$ & $\ldots$ & $\ldots$ & 0 \\
$\vdots$ & $\vdots$ & $\vdots$ & $\vdots$ & $\vdots$ & $\vdots$ & $\vdots$ & $\vdots$ & $\vdots$ & $\vdots$ & $\vdots$ & $\vdots$ & $\vdots$ & $\vdots$ & $\vdots$ & $\vdots$ & $\vdots$ \\
& & & & & & & & & & & & & & & & $\vdots$ \\
$\vdots$ & $\vdots$ & $\vdots$ & $\vdots$ & $\vdots$ & $\vdots$ & $\vdots$ & $\vdots$ & $\vdots$ & $\vdots$ & $\vdots$ & $\vdots$ & $\vdots$ & $\vdots$ & $\vdots$ & $\vdots$ & $\vdots$ \\
$\vdots$ & $\vdots$ & $\vdots$ & $\vdots$ & $\vdots$ & $\vdots$ & $\vdots$ & $\vdots$ & $\vdots$ & $\vdots$ & $\vdots$ & $\vdots$ & $\vdots$ & $\vdots$ & $\vdots$ & $\vdots$ & $\vdots$ \\
0 & 0 & 0 & $\ldots$ & $\ldots$ & $\ldots$ & $\ldots$ & $\ldots$ & $\ldots$ & $\ldots$ & $\ldots$ & $\ldots$ & 0 & $a$ & $b$ & $a$ & 0
\end{tabular}

The A-polynomial of $M_{4 k+2}$ is a factor of the above $(6 k+2) \times(6 k+2)$ determinant, where in the determinant, $\sigma=1+2 p^{2}+p^{4}, a=q p^{2}+p^{4} q^{2}-q-p^{2} q^{2}, b=-p^{4} q^{3}+q^{2}-2 p^{2} q^{2}+2 q p^{2}-p^{4} q+1$. 
It looks fairly difficult to calculate this determinant. In Section 1, we have shown that the Newton polygon of the $A$-polynomial of $M_{4 k+2}$ must have an edge of slope $k$. We notice that one can calculate directly this edge polynomial, without knowing explicit expression of the $A$-polynomial. The procedure is as follows. First we make a change of bases: from the standard basis $\left(\mu_{2}, \lambda_{2}\right)$ to $\left(\lambda_{2}, \beta_{2}=\mu_{2} \lambda_{2}^{k}\right)$. Let $w$ be the eigenvalue of $\rho\left(\beta_{2}\right)$. Then the $A$-polynomial of $M_{4 k+2}$ with respect to the new basis $\left\{\lambda_{2}, \beta_{2}\right\}$ can be obtained from the old one by making the simple substitution $p=\frac{w}{q^{k}}$ (up to a multiple of a certain power of $q$ ). The new $A$-polynomial will be in variables $q$ and $w$ and the Newton polygon of this polynomial will have an edge of slope $1 / 0$ in the $(q, w)$-plane. So if we simply let $q=0$ in the new $A$-polynomial, then the resulting polynomial in $w$ must contain a factor which is the required edge polynomial. Also this factor cannot be just a power of $w$ (i.e. it contains at least two terms). Now we give the detailed calculation.

In the old determinant expression above (which formally contains the $A$-polynomial of $M_{4 k+2}$ as a factor), let $p=\frac{w}{q^{k}}$. Then the resulting polynomial in variables $q$ and $w$ should contain the new $A$-polynomial of $M_{4 k+2}$ (with respect to the basis $\left\{\lambda_{2}, \beta_{2}\right\}$ ) as a factor (up to a multiple of power of $q$ ). Now we need to find out all the terms in the standard expansion of the new determinant which have the lowest power in $q$ (a negative power). Say, the power is $-m$ for some positive integer $m$. If the sum of these terms is not zero, then the product of $q^{m}$ and the determinant is a polynomial in $\mathbb{Z}[q, w]$ which contains, when valued at $q=0$, the edge polynomial we are seeking as a factor. So to find such terms, we may replace $\sigma, a$ and $b$ in the old determinant above only by $\left(\frac{w}{q^{k}}\right)^{4}, \frac{w^{4}}{q^{4 k-2}}$ and $-\frac{w^{4}}{q^{4 k-1}}$ respectively. Then the determinant becomes (see next page) 


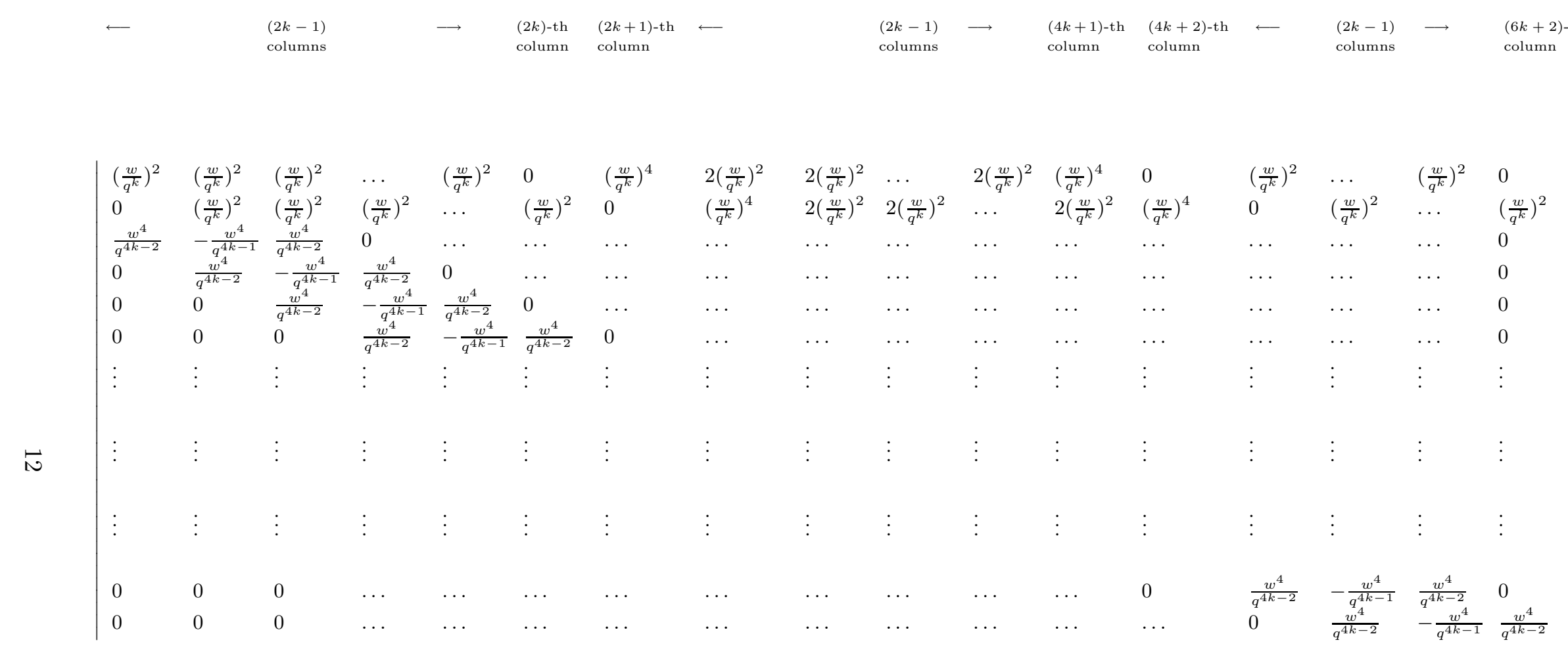

This determinant is a rational polynomial in $w$ and $q$ which can be simplified as $\Sigma_{i=-m}^{i=n} C_{i}(w) q^{i}$, where $n>m>0$ are integers and $C_{i}(w)$ are polynomials in $w$. $C_{-m}(w)$ contains a factor which is the edge polynomial of $M_{4 k+2}$. 
The standard expansion of the above determinant consists of the sum over all possible terms, where each term is a product of entries one from each row and each column. Appropriate sign factors have to be included. Through a series of observations, we now try to determine all the terms in the expansion of this determinant which have the highest power of $q$ in the denominator.

The first and the last columns each have only 2 non-zero entries. Name these entries as follows : $(1,1)$ entry as $A,(3,1)$ entry as $B,(2,6 k+2)$ entry as $C$ and $(6 k+2,6 k+2)$ entry as $D$. Then every non-zero term in the expansion of the above determinant has one of the following four factors: $A C, A D, B C$ or $B D$. This leads to the following four cases.

Case $A C$. Of all the terms which contain $A C$ in the expansion of the determinant, the term which is the product of $A$ and $C$ and the entries $(3,2),(4,3), \ldots,(6 k+2,6 k+1)$ gives the maximal power of $q$ in the denominator. The total power of $q$ of this term is $24 k^{2}-2 k$. The corresponding power of $w$ is $24 k+4$. That is, this term is equal to $\frac{w^{24 k+4}}{q^{24 k^{2}-2 k}}$.

Case $A D$. Of all the terms which contain $A D$ in the expansion of the determinant, the term which is the product of $A, D$, the entry $(2,4 k+2)$ with the entries $(3,2),(4,3),(5,4), \ldots,(4 k+$ $2,4 k+1)$ along with the entries $(4 k+3,4 k+3),(4 k+4,4 k+4), \ldots,(6 k, 6 k),(6 k+1,6 k+1)$ gives the maximal power of $q$ in the denominator. This term is equal to $\frac{w^{24 k+6}}{q^{24 k^{2}-2 k}}$.

Case $B C$. Of all the terms which contain $B C$ in the expansion of the determinant, the term which is the product of $B, C$, the entry $(1,2 k+1)$ with the entries $(4,2),(5,3), \ldots,(2 k+$ $1,2 k-1),(2 k+2,2 k)$ along with the entries $(2 k+3,2 k+2),(2 k+4,2 k+3), \ldots,(6 k+2,6 k+1)$ gives the maximal power of $q$ in the denominator. This term is equal to $\frac{w^{24 k+6}}{q^{24 k^{2}-2 k}}$.

Case $B D$. Of all the terms which contain $B D$ in the expansion of the determinant, the term which is the product of $B, D$, the entries $(1,2 k+1)$ and $(2,4 k+2)$, with the entries $(4,2),(5,3), \ldots,(2 k+1,2 k-1),(2 k+2,2 k)$, with the entries $(2 k+3,2 k+2),(2 k+4,2 k+$ $3) \ldots(4 k+1,4 k),(4 k+2,4 k+1)$ along with the entries $(4 k+3,4 k+3), \ldots,(6 k, 6 k),(6 k+$ $1,6 k+1)$ gives the maximal power of $q$ in the denominator. This term is equal to $\frac{w^{24 k+8}}{q^{24 k^{2}-2 k}}$. In calculating the terms above, we have taken into consideration of the signs of these terms; they all have positive sign. From the above four cases, the negative highest possible power of $q$ among all terms in the expansion of the determinant is $-\left(24 k^{2}-2 k\right)$. We have four such terms. Their numerators sum up to $w^{24 k+4}\left(1+w^{2}+w^{2}+w^{4}\right)=w^{24 k+4}\left(w^{2}+1\right)^{2}$. As we noted already, if we multiply the whole determinant by $q^{24 k^{2}-2 k}$ we get a polynomial $P(q, w)$ in $\mathbb{Z}[w, q]$ which has no $q$ as a factor and which contains the $A$-polynomial of $M_{4 k+2}$ as a factor, and further if we set $q=0$ in $P(q, w)$, we get the polynomial $w^{24 k+4}\left(w^{2}+1\right)^{2}$ which should contain the edge polynomial, which we are seeking, as a factor. Obviously $w^{2}+1$ must be a factor of the edge polynomial. This polynomial has a root of unity of 
order 4. Now the conclusion of Theorem 1 follows from property (3) of an edge polynomial listed in Section 1, noticing that the slope of the edge we have now in the $q w$-plane is 1/0.

\section{References}

[CCGLS] D. Cooper, M. Culler, H. Gillet, D. Long, and P. Shalen, Plane curves associated to character varieties of 3-manifolds, Invent. Math. 118 (1994), 47-84.

[CL] D. Cooper and D. Long, Remarks on the A-polynomial of a knot, J. Knot Theory and Its Ramifications, 5 (1996) 609-628.

[CJR] M. Culler, W. Jaco and H. Rubinstein, Incompressible surfaces in once-punctured torus bundles. Proc. London Math. Soc. (3) 45 (1982) 385-419.

[CS] M. Culler and P. Shalen, Varieties of group representations and splittings of 3-manifolds, Ann. of Math. 117 (1983) 109-146.

[D] N. Dunfield, Examples of non-trivial roots of unity at ideal points of hyperbolic 3manifolds. Topology 38 (1999) 457-465.

[FH] W. Floyd and A. Hatcher, Incompressible surfaces in punctured-torus bundles, Topology Appl. 13 (1982) 263-282.

[HMW] C. Hodgson, R. Meyerhoff and J. Weeks, Surgeries on the Whitehead link yield geometrically similar manifolds, Topology'90, (1992) 195-206..

[O] T. Ohtsuki, Ideal points and incompressible surfaces in two-bridge knot complements, J. Math. Soc. Japan 46 (1994) 51-87.

Department of Mathematics, SUNY at Buffalo, Buffalo, NY 14260-2900

kuppum@acsu.buffalo.edu

xinzhang@math.buffalo.edu 